\title{
The importance of carotid artery stiffness and increased intima- media thickness in obese children
}

\author{
Mustafa Ozcetin, Z Ruken Yuksekkaya Celikyay, Atac Celik, Resul Yilmaz, Yasemin Yerli, Unal Erkorkmaz
}

Background. Atherosclerosis that starts in childhood invariably advances during adulthood.

Aim. We aimed to study the effect of obesity on main carotid artery intima-media thickness (CIMT) and arterial stiffness.

Material and methods. A total of 78 children were studied from October 2010 to February 2011. They were divided into obese $(n=42$, group 1) and normal ( $n=36$, group 2). All children were subjected to physical examination, routine biochemical and haematological analysis, carotid ultrasonography and echocardiographic measurements. A detailed medical history was obtained. Body mass index (BMI) was calculated by dividing participants' weight in kilograms by the square of their height in metres. Stiffness index $\beta$ was calculated using blood pressure and diameter of the systolic and diastolic artery. Intima-media thickness was also measured.

Results. The mean age of the obese and normal (control) groups was $10.12 \pm 2.12$ years and $9.78 \pm 1.78$ years, respectively. Weight, BMI, and systolic and diastolic blood pressure values were significantly higher in the obese group (all $p<0.001$ ). In terms of arterial stiffness and CIMT measurements, all parameters were higher in the obese group than the control $(p<0.001)$. There was a relationship between the degree of obesity and CIMT or stiffness index $\beta$. In addition, dilatation and hypertrophy levels in the left ventricle were higher in obese children.

Conclusions. Obese children with risk factors for multiple atherosclerosis could have increased CIMT dimensions and, consequently, should be screened for these risks. Ultrasonographic CIMT and arterial stiffness measurements can detect vascular damage at an early stage of development in children with cardiovascular risk factors.

S Afr Med J 2012;102(5):295-299.
Childhood obesity is a major health problem throughout the world Obese children are more likely to become obese adults in whom one can expect a higher rate of hospitalisation, interventions and premature death. ${ }^{1}$ In obese people, coronary heart disease generally manifests in middle age or later life. However, atherosclerosis has its roots in childhood, its first signs in obese children appearing before puberty; therefore, it is important to develop efficient strategies to reduce the incidence of cardiovascular disease (CVD) in the population. Carotid artery intima-media thickness (CIMT) serves as a marker of preclinical atherosclerosis. ${ }^{2}$

CVD develops as a result of arterial damage in the form of arteriosclerosis. Arteriosclerosis is a general term for conditions in which diffuse thickening and stiffening in mainly large- and

Department of Pediatrics, Zonguldak Karaelmas University School of Medicine, Zonguldak, Turkey

Mustafa Ozcetin, MD

Department of Radiology, Gaziosmanpasa University School of Medicine, Tokat Turkey

Z Ruken Yuksekkaya Celikya, MD

Department of Cardiology, Gaziosmanpasa University School of Medicine, Tokat Turkey

Atac Celik, MD

Department of Pediatrics, Gaziosmanpasa University School of Medicine, Tokat, Turkey

Resul Yilmaz, MD

Yasemin Yerli, MD

Department of Medical Statistics, Gaziosmanpasa University School of Medicine, Tokat, Turkey

Unal Erkorkmaz, $\mathrm{PhD}$ medium-sized arteries develop under different conditions. Both the media and intima of the arteries could be involved in the pathology. Risk factors diagnosed in childhood can predict the development of preclinical carotid atherosclerosis in adults. ${ }^{2}$ However, there is limited information about early atherosclerosis and its mechanisms in childhood.

Many studies mention close relationships between traditional anthropometric, haemodynamic and biochemical cardiovascular risk factors during childhood and adolescence, and high rates of CIMT and vascular events in the 40s. Importantly, exposure to cardiovascular risk factors is associated with vascular damage. In addition, because of the cumulative nature of risk factors, obesity and central fat distribution in childhood can provide clues about CIMT in adulthood. ${ }^{3}$

We aimed to study the effect of obesity on main carotid artery lumen diameter and intima-media thickness and the relationships with the early stages of atherosclerosis.

\section{Material and methods Patients}

This was a prospective case-control study from October 2010 to February 2011 on schoolchildren aged 8 - 16 years. Obese children were transferred from a primary healthcare paediatrician to our paediatric outpatient clinic in Gaziosmanpasa University Hospital. Patients with dysmorphic syndromes and/or endocrine disorders were excluded. A control group of children of similar age and gender distribution without obesity and dyslipidaemia was included in the study. None of the children was on cardiovascular medication, and all were non-smokers. Children were included only after obtaining informed consent from their guardians. The study protocol was approved by the Committee on Ethical Practice of the hospital.

\section{Clinical evaluation}

For each patient, data about family and personal history for cardiovascular events, growth curve and nutritional assessment were recorded. Height, weight and blood pressure (BP) were measured. 
Body mass index (BMI) was calculated as participant's weight in $\mathrm{kg}$ divided by the square of their height in metres. Obesity was defined as a BMI greater than the 95th percentile for age, based on gender and population-specific data. BP was measured in children using a validated protocol. Systolic blood pressure (SBP) and diastolic blood pressure (DBP) were measured twice, based on previously reported methods. Hypertension was defined as BP values above the 95th percentile for height, age and gender.

\section{Biochemical analysis}

For glucose, triglyceride (TG), total cholesterol (TC), high-density lipoprotein cholesterol (HDL-C) and low-density lipoprotein cholesterol (LDL-C) levels, blood samples were obtained after a 12-hour fast. Plasma concentrations of TC and TG were measured using routine enzymatic methods, and HDL-C was measured using a homogeneous colorimetric method. LDL-C was calculated using the formula by Friedewald.

\section{Carotid ultrasonography}

Ultrasonic examinations of the stiffness index $\beta$ of the common carotid arteries were carried out in the supine position with slight hyperextension of the neck, using a high-resolution ultrasonic phaselocked echo-tracking system. The stiffness index $\beta$, which refers to elasticity of the arterial wall, was calculated using BP and the diameter of the artery based on the following formula:

\section{Stiffness index $\beta=[\ln (\mathrm{Ps} / \mathrm{Pd})] \times \mathrm{Dd} /(\mathrm{Ds}-\mathrm{Dd})$}

where Ps and Pd are the systolic and diastolic blood pressures, and Ds and Dd are the systolic and diastolic inner diameters of the artery, respectively. The stiffness index $\beta$ is a quantity adjusted for blood pressure.

\section{Echocardiographic measurements}

All the subjects were evaluated for echocardiographic features (2.5 $\mathrm{mHz}$ transducer, Philips EnVisor C, Bothell, Washington, USA). Cardiac anatomy, ventricular function and valve competence were assessed using standardised projections and measurements. Simpson's method was used to measure left ventricular ejection fraction (LVEF). Left ventricular mass was determined by the formula given by Devereux et al., and the left ventricular mass index was calculated by dividing the left ventricular mass by the body surface area. ${ }^{4}$

The following conventional mitral inflow pulse wave Doppler parameters were measured: peak velocity of early diastolic filling (E), late filling (A), and deceleration time (DT) of the E-wave velocity. These parameters were obtained from the apical four-chamber view with a $1-3 \mathrm{~mm}$ sample volume placed between the mitral leaflet tips during diastole. Pulmonary venous flow parameters were also measured: peak systolic velocity (Ps), peak anterograd diastolic velocity (Pd) and the Ps/Pd ratio. These parameters were obtained from the apical four-chamber view with a $2-3 \mathrm{~mm}$ sample volume placed $1 \mathrm{~cm}$ into the pulmonary vein. Tissue Doppler parameters were measured: early diastolic mitral annular velocity $(\mathrm{Em})$ and late diastolic mitral velocity (Am). These parameters were obtained from the apical four-chamber view with a $2-5 \mathrm{~mm}$ sample volume placed $1 \mathrm{~cm}$ within the septal and lateral insertions of the mitral leaflets. The average value from $\geq 3$ measurements was used for analysis of the Doppler data. The ratio of mitral peak velocity of early diastolic filling to early diastolic mitral annular velocity $(\mathrm{E} / \mathrm{Em})$ was calculated for the lateral and septal annulus, and the mean of the lateral and septal $\mathrm{E} / \mathrm{Em}$ was also determined.

\section{Statistical analysis}

According to the Kolmogorov-Smirnov normality test, two independent sample $t$-tests were used to compare the normally distributed independent variables between the two groups, and the Mann-Whitney U-test was used to compare the non-normally distributed independent variables. Normally distributed continuous data were expressed as mean \pm standard deviation (SD); non-normally distributed continuous variables were presented as median and interquartile range (IQR) (quartile 1 - quartile 3). For comparing with categorical data, a chisquare test was used. Categorical data were expressed as numbers and percentages. $p$-values $>0.05$ were considered statistically significant. Statistical analyses were performed using SPSS software (IBM SPSS Statistics 19, SPSS Inc., Somers, NY).

\section{Results}

The study group included a total of 78 children aged 8 - 16 years, including 32 (41\%) boys and 46 (59\%) girls, divided into two groups: (i) a patient group of 42 obese children and (ii) a control group of 36 normal-weight children. Age and gender percentages of the two groups were similar $(p>0.05)$. The average age was $10.12 \pm 2.12$ years in the obese group, and $9.78 \pm 1.78$ years in the control group. BMI values of the obese and control groups were $27.83 \pm 2.53 \mathrm{~kg} / \mathrm{m}^{2}$ and $19.03 \pm 2.54 \mathrm{~kg} / \mathrm{m}^{2}$, respectively. The weight, height and BMI of the two groups differed significantly. Weight and BMI values were significantly higher in the obese group $(p<0.001)$, which also had significantly higher SBP and DBP values (all $p<0.001$ ), but SBP values higher than the 95th percentile were recorded for only 2 subjects in the obese group. None of the children in the control group had SBP and DBP values above the 95th percentile (Table 1). Table 2 gives the results of some clinical tests. There was no difference between the two groups for TC, LDL-C and TG ( $p>0.05$ ) (Table 2).

All parameters of arterial stiffness and CIMT were higher in obese patients than the controls $(p<0.001)$. There were also significant differences between obese and controls for carotid artery diastolic diameter (6.8 and $6.1 \mathrm{~mm}$, respectively; $p<0.001)$ and for systolic diameter ( 7.6 and $7.0 \mathrm{~mm}$, respectively; $p<0.001)$. Carotid artery systolic and diastolic diameters were significantly higher in the obese group than the control $(p<0.001)$ (Table 1). No atherosclerotic plaques were found in any patient. There were significant correlations between CIMT and BMI or SBP. Stiffness index $\beta$, on the other hand, had significant correlations with BMI, SBP and DBP. There were close associations between degree of obesity and CIMT and stiffness index $\beta$ (Table 3 ).

Obese and control groups did not differ in terms of left ventricular ejection fraction, end-systolic dimension, and mass index (Table 4). However, the obese group had higher blood pressure and hypertrophy of the left ventricle and higher left atrial, left venricular and aortic root size (Tables 1 and 4). However, hypertrophy in the left ventricle was not significant, based on mass index values. Conventional Doppler parameters did not differ between the two groups. Doppler parameters measured from the lateral mitral annulus were significantly different while mean E/Em ratio was not (Table 4).

\section{Discussion}

Modern lifestyle changes including new eating habits, reduced physical activity and more time spent on television and computer games, have conduced to obesity in childhood and adolescence. About a third of obese children and $80 \%$ of obese adolescents remain obese as adults. ${ }^{5}$ It is known that atherogenic development from paediatric age favours cardiovascular problems. The increasing prevalence worldwide of overweight and obesity are the most important. ${ }^{1}$ If detected early, atherosclerosis is reversible and suitable precautions can be taken. ${ }^{6}$ To demonstrate an increase in 
Table 1. Baseline and vascular observations of patient and control groups

\begin{tabular}{|c|c|c|c|}
\hline & Control group $(n=36)$ & Obese group $(n=42)$ & $p$-values \\
\hline Gender (male) & $14(38.9 \%)$ & $18(42.9 \%)$ & 0.901 \\
\hline Mean age (years) & $9.78 \pm 1.78$ & $10.12 \pm 2.12$ & 0.462 \\
\hline Weight (kg) & $37.36 \pm 9.65$ & $61.52 \pm 14.12$ & $<0.001^{\star}$ \\
\hline Height $(\mathrm{cm})$ & $139.61 \pm 10.92$ & $147.86 \pm 13.15$ & $0.004^{*}$ \\
\hline BMI $\left(\mathrm{kg} / \mathrm{m}^{2}\right)$ & $19.03 \pm 2.54$ & $27.83 \pm 2.53$ & $<0.001^{\star}$ \\
\hline SBP $(\mathrm{mmHg})$ & $90.00[90.00-95.00]$ & $110.00[100.00-115.00]$ & $<0.001^{\dagger}$ \\
\hline DBP (mmHg) & $60.00[60.00-65.00]$ & $65.00[60.00-70.00]$ & $<0.001^{\dagger}$ \\
\hline CIMT (mm) & $0.48 \pm 0.72$ & $0.56 \pm 0.07$ & $<0.001^{\star}$ \\
\hline Stiffness index $\beta$ & $2.80 \pm 0.65$ & $4.29 \pm 1.27$ & $<0.001^{\star}$ \\
\hline Ds (mm) & $7.00 \pm 0.62$ & $7.60 \pm 0.61$ & $<0.001^{\star}$ \\
\hline $\mathrm{Dd}(\mathrm{mm})$ & $6.10 \pm 0.64$ & $6.80 \pm 0.53$ & $<0.001^{*}$ \\
\hline
\end{tabular}

Table 2. Laboratory characteristics of patient and control groups

\begin{tabular}{llll}
\hline & Control group $(\boldsymbol{n}=\mathbf{3 6})$ & Obese group $(\boldsymbol{n}=\mathbf{4 2})$ & $\boldsymbol{p}$-values \\
TC $(\mathrm{mg} / \mathrm{dl})$ & $159.57 \pm 18.18$ & $165.35 \pm 30.28$ & 0.629 \\
TG $(\mathrm{mg} / \mathrm{dl})$ & $91.57 \pm 23.27$ & $144.51 \pm 81.89$ & 0.099 \\
LDL-C $(\mathrm{mg} / \mathrm{dl})$ & $91.29 \pm 12.77$ & $101.57 \pm 25.00$ & 0.122 \\
HDL-C $(\mathrm{mg} / \mathrm{dl})$ & $56.43 \pm 13.19$ & $49.61 \pm 11.19$ & 0.156 \\
Glucose $(\mathrm{mg} / \mathrm{dl})$ & $86.00 \pm 4.24$ & $89.00 \pm 6.31$ & 0.746 \\
& & &
\end{tabular}

Table 3. Spearman's correlation coefficients

\begin{tabular}{|c|c|c|c|}
\hline & BMI & SBP & DBP \\
\hline $\operatorname{CIMT}(N=78)$ & $0.321^{\star}$ & $0.306^{*}$ & 0.205 \\
\hline Stiffness index $\beta(N=78)$ & $0.401^{*}$ & $0.629^{*}$ & $0.253^{*}$ \\
\hline \multicolumn{4}{|c|}{$\begin{array}{l}\text { *statistically significant correlations }(\mathrm{p}<0.05) . \\
\text { BMI = body mass index; CIMT = carotid intima-media thickness; SBP = systolic blood pres- } \\
\text { sure; DBP = diastolic blood pressure. } \\
\text { Table contents are Spearman's rank correlation coefficients. }\end{array}$} \\
\hline
\end{tabular}

IMT, arterial stiffness and endothelial dysfunction at the carotid artery, high resolution B-mode ultrasonography is used. ${ }^{6}$

Some studies have focused on the relationship between childhood BMI and BP and CIMT as risk factors for CVD. For the assessment of subclinical atherosclerosis, CIMT that is associated with coronary artery disease, stroke and deterioration of coronary atherosclerosis can be used as a non-invasive tool. ${ }^{7}$ Higher CIMT is an established predictor for early preclinical atherosclerosis. However, the factors for premature carotid atherosclerosis in obese children have not been fully elucidated. Recent studies on obese children indicated significantly higher CIMT. ${ }^{2}$ In contrast to arterial wall thickening, arterial stiffness is a functional change in atherosclerosis. The stiffness index $\beta$ determined by ultrasonographic observation refers to the elasticity of the arterial wall, which is in turn associated with an increase in collagen content and a decrease in elastin content owing to pathological changes. Many studies also report associations between stiffness index $\beta$ and peripheral artery disease. ${ }^{8}$
We found that CIMT was significantly higher in the obese than the control group, in keeping with previous studies. ${ }^{2}$ Two studies ${ }^{9,10}$ dealing with normotensive obese children reported no difference between obese and normal groups for CIMT. Aggoun et al. ${ }^{11}$ reported that there was no significant increase in CIMT in prepubescent obese children. Half of these children had ambulatory systolic hypertension. Morrison et al. ${ }^{12}$ observed a clustering of cardiovascular risk factors (modified National Cholesterol Education Program (NCEP) criteria) in a minority of overweight and obese children where CIMT was slightly, but not significantly, higher in the patient group. Furthermore, CIMT levels between obese with clustering and those without were not different.

Positive correlations were reported between childhood BMI and SBP and DBP, while others showed a positive relationship with SBP only. Sorof et al. ${ }^{13}$ demonstrated that CIMT was higher in hypertensive children than in normotensive subjects, and that only BMI was independently associated with CIMT. In our study, obese children had higher SBP and DBP values than those in the control group. Increased CIMT and stiffness index $\beta$ had significant correlations with the degree of obesity. Moreover, BMI and SBP had significant correlations with CIMT and arterial stiffness. Elevated CIMT may be a result of non-atherosclerotic changes in the arterial wall. Ultrasonographic methods for measuring CIMT cannot distinguish intimal atherosclerosis from medial hypertrophy owing to pressure effects. ${ }^{13}$ We also observed that CIMT is associated with $\mathrm{BMI}$ and SBP.

Obese children are more likely to remain obese in adulthood. Childhood obesity is an important indicator for CVDs in adult life. ${ }^{14}$ 
Table 4. Echocardiographic parameters of patient and control groups

\begin{tabular}{|c|c|c|c|}
\hline & Control group $(n=36)$ & Obese group $(n=42)$ & $p$-values \\
\hline $\operatorname{LVEF}(\%)$ & $67.79 \pm 5.17$ & $67.81 \pm 5.82$ & 0.992 \\
\hline LVEDD (mm) & $39.21 \pm 3.53$ & $41.38 \pm 4.24$ & 0.034 \\
\hline $\operatorname{LVESD}(\mathrm{mm})$ & $24.71 \pm 2.29$ & $25.54 \pm 2.52$ & 0.187 \\
\hline IVSD (mm) & $8.06 \pm 1.25$ & $9.12 \pm 1.14$ & 0.001 \\
\hline Aortic root diameter (mm) & $20.47 \pm 2.21$ & $22.92 \pm 2.19$ & $<0.001$ \\
\hline LVPW (mm) & $7.00[6.00-7.00]$ & $8.00[7.00-9.00]$ & $<0.001$ \\
\hline Left atrium diameter $(\mathrm{mm})$ & $27.26 \pm 2.83$ & $30.88 \pm 3.40$ & $<0.001$ \\
\hline Left ventricular mass index $\left(\mathrm{gr} / \mathrm{m}^{2}\right)$ & $69.79 \pm 11.21$ & $70.58 \pm 12.27$ & 0.798 \\
\hline Ps & $58.47 \pm 10.94$ & $56.31 \pm 9.65$ & 0.428 \\
\hline $\mathrm{Pd}$ & $61.12 \pm 12.63$ & $52.12 \pm 8.13$ & 0.001 \\
\hline $\mathrm{Ps} / \mathrm{Pd}$ & $1.00 \pm 0.30$ & $1.10 \pm 0.20$ & 0.152 \\
\hline $\mathrm{E}(\mathrm{cm} / \mathrm{sec})$ & $106.21 \pm 16.31$ & $105.69 \pm 14.04$ & 0.898 \\
\hline $\mathrm{A}(\mathrm{cm} / \mathrm{sec})$ & $68.21 \pm 13.60$ & $70.00 \pm 10.32$ & 0.577 \\
\hline $\mathrm{E} / \mathrm{A}$ & $1.61 \pm 0.43$ & $1.54 \pm 0.30$ & 0.454 \\
\hline \multicolumn{4}{|l|}{ Lateral } \\
\hline $\operatorname{Em}(\mathrm{cm} / \mathrm{sec})$ & $21.09 \pm 3.33$ & $19.32 \pm 3.46$ & 0.052 \\
\hline $\operatorname{Am}(\mathrm{cm} / \mathrm{sec})$ & $8.21 \pm 1.61$ & $9.12 \pm 1.42$ & 0.028 \\
\hline $\mathrm{Em} / \mathrm{Am}$ & $2.64 \pm 0.53$ & $2.19 \pm 0.60$ & 0.003 \\
\hline $\mathrm{E} / \mathrm{Em}$ & $2.29 \pm 0.82$ & $2.91 \pm 1.27$ & 0.039 \\
\hline \multicolumn{4}{|l|}{ Septal } \\
\hline $\operatorname{Em}(\mathrm{cm} / \mathrm{sec})$ & $14.82 \pm 1.75$ & $14.00 \pm 2.20$ & 0.115 \\
\hline $\operatorname{Am}(\mathrm{cm} / \mathrm{sec})$ & $8.15 \pm 1.91$ & $8.48 \pm 1.66$ & 0.488 \\
\hline $\mathrm{Em} / \mathrm{Am}$ & $1.90 \pm 0.47$ & $1.71 \pm 0.41$ & 0.097 \\
\hline $\mathrm{E} / \mathrm{Em}$ & $3.24 \pm 1.21$ & $4.00 \pm 1.80$ & 0.075 \\
\hline \multicolumn{4}{|c|}{$\begin{array}{l}\mathrm{LVEF}=\text { left ventricular ejection fraction; } \mathrm{LVEDD}=\text { left ventricular end-diastolic dimension; } \mathrm{LVESD}=\text { left ventricular end-systolic dimension; } \mathrm{IVSD}=\text { interventricular septal dimension; } \mathrm{LVPW}= \\
\text { left ventricular posterior wall; } \mathrm{P} / \mathrm{Pd}=\text { ratio of peak systolic velocity and peak anterograd diastolic velocity of pulmonary venous flow; } \mathrm{E}=\text { peak velocity of early diastolic filling; } \mathrm{A}=\text { peak velocity of } \\
\text { late filling; } \mathrm{Em}=\text { early diastolic mitral annular velocity; } \mathrm{Am}=\text { late diastolic mitral annular velocity; } \mathrm{E} / \mathrm{Em}=\text { early mitral inflow velocity to early diastolic mitral annular velocity ratio. } \\
\text { Data are shown as mean } \pm \mathrm{SD} \text {, median [interquartile range]. }\end{array}$} \\
\hline
\end{tabular}

Obesity may increase left ventricular dilatation by increasing left ventricular filling pressure and volume. Increased ventricular wall stress is stabilised with an increase in myocontractile structures, which in turn results in ventricular hypertrophy. ${ }^{15}$ Left ventricular hypertrophy is also a predictor for various CVDs in early life. ${ }^{4}$ Mahoney et al. ${ }^{16}$ concluded that there was an increase in left ventricular mass even with minor BP increases. In our study, obese children had more dilated and hypertrophied left ventricules. Higher BP in the obese group could explain this dilatation and hypertrophy. Moreover, the more dilated left atrium and aortic root in obese subjects support this hypothesis. The small sample size may be a reason for the lack of significant differences between the two groups for left ventricular mass index. It is logical to expect diastolic dysfunctionality in a group with higher BP and more dilated and hypertrophied ventricules. However, there were no obvious deteriorations in diastolic functions in obese children. Small sample size could be a possible explanation for this finding.

There are limitations in our study. CIMT was measured at the common carotid arteries. However, IMT measurements in the common carotid artery are more reliable than IMT measurements in the carotid bifurcation or in the internal carotid artery. It is also less sensitive to local atherosclerotic changes. We only looked at the common CIMT and did not examine the intima-media thicknesses in the carotid bulb or the internal carotid artery; the evaluation of atherosclerosis in these segments may vary. CIMT is thought to increase slowly over time. Increases in CIMT and arterial stiffness index in obese children with increasing BMI in our study are similar to others in the literature.

\section{Conclusion}

Obese children with multiple atherosclerosis-promoting risk factors may especially have higher CIMT and consequently should be screened for these risks. The use of carotid artery ultrasound may help to further the identification of children at high risk of developing earlier atherosclerosis. Owing to the rarity of cardiovascular clinical events in childhood, additional cross-sectional, rather than longitudinal, studies with standardised CIMT measurement methods are needed to define reliable normal values based on age and gender in the paediatric population.

\section{References}

1. Lobstein T, Baur L, Uauy R. International Obesity Task Force. Obesity in children and young people: a crisis in public health. Obes Rev 2004;5:4-85.

2. Lorenz MW, Markus HS, Bots ML, Rosvall M, Sitzer M. Prediction of clinical cardiovascular events with carotid intima-media thickness: a systematic review and meta-analysis. Circulation 2007;115:459-

3. Johnson HM, Douglas PS, Srinivasan SR, et al. Predictors of carotid intima-media thickness progression in young adults. The Bogalusa heart study. Stroke 2007;38:900-905

4. Devereux RB, Alonso DR, Lutas EM, et al. Echocardiographic assessment of left ventricular hypertrophy: comparison to necropsy findings. Am J Cardiol 1986;57:450-458.

5. Alemzadeh R, Lifshitz F. Childhood Obesity. In: Lifshitz F, ed. Pediatric Endocrinology, 4th ed. New York: Marcel Dekker, 2003:823-858. 


\section{RESEARCH}

6. De Groot E, Hovingh GK, Wiegman A, et al. Measurement of arterial wall thickness as a surrogate marker for atherosclerosis. Circulation 2004;109:1133-1138.

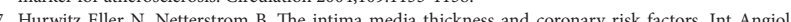
2001;20:118-125.

8. Núñez F, Martínez-Costa C, Sánchez-Zahonero J. Carotid artery stiffness as an early marker of vascular lesions in children and adolescents with cardiovascular risk factors. Rev Esp Cardiol 2010;63:1253-1260. 9. Di SG, Pacileo G, Del Giudice EM, et al. Abnormal myocardial deformation properties in obese, nonhypertensive children: an ambulatory blood pressure monitoring, standard echocardiographic, and strain rate imaging study. Eur Heart J 2006;27:2689-2695.

10. Tounian P, Aggoun Y, Dubern B, et al. Presence of increased stiffness of the common carotid artery and endothelial dysfunction in severely obese children: a prospective study. Lancet 2001;358:1400-1404.

11. Aggoun Y, Farpour-Lambert NJ, Marchand LM, et al. Impaired endothelial and smooth muscle functions and arterial stiffness appear before puberty in obese children and are associated with elevated ambulatory blood pressure. Eur Heart J 2008;29:792-799.

12. Morrison KM, Dyal L, Conner W, et al Cardiovascular risk factors and non-invasive assessment of subclinical atherosclerosis in youth. Atherosclerosis 2010;208:501-505.

13. Sorof JM, Alexandrov AV, Cardwell G, Portman RJ. Carotid artery intimamedial thickness and left ventricular hypertrophy in children with elevated blood pressure. Pediatrics 2003;111:61-66.
ver
14. Steinberger J, Daniels SR, Eckel RH, et al. Progress and challenges in metabolic syndrome in children and adolescents: a scientific statement from the American Heart Association Atherosclerosis, Hypertension, and Obesity in the Young Committee of the Council on Cardiovascular Disease in the Young; Council on Cardiovascular Nursing; and Council on Nutrition, Physical Activity, an Metabolism. Circulation 2009,119.628-647.

5. Poirie $\mathrm{P}$, Gles ID, Bray GA, et al. Obesity and cardiovascular disease: pathophysiology, evaluation and effect of weight loss: an update of the 1997 American Heart Association Scientific Statement o Obesity and Heart Disease from the Obesity Committee of the Council on Nutrition, Physical Activity, and Metabolism. Circulation 2006;113:898-918.

6. Mahoney LT, Schieken RM, Clarke WR, Lauer RM. Left ventricular mass and exercise responses predict future blood pressure. The Muscatine Study. Hypertension 1988;12:206-213.

Accepted 22 December 2011 